\title{
Dispersion and stabilization of TiN and TiC nanoparticles in organic suspensions
}

\author{
C. Mendoza ${ }^{a,}{ }^{*}$, Z. González ${ }^{b}$, Y. Castro ${ }^{c}$ and B. Ferrari ${ }^{d}$ \\ ${ }^{1}$ Instituto de Cerámica y Vidrio. CSIC. c/ Kelsen, 5. Madrid. Spain \\ acmendoza@icv.csic.es, ${ }^{\mathrm{b}} \mathrm{z}$ gonzalez@icv.csic.es, ${ }^{\mathrm{c}} \mathrm{castro@icv.csic.es,}{ }^{\mathrm{d} b f e r r a r i @ i c v . c s i c . e s}$
}

Keywords: TiN; TiC; Colloidal; Electrophoretic deposition; Hydrazine.

\begin{abstract}
In this work the stability of TiN and $\mathrm{TiC}$ nanopowders in isopropyl alcohol as well as the fabrication of dense and well adhered thin coatings based on TiN and $\mathrm{TiC}$ by electrophoretic deposition (EPD) have been evaluated in terms of zeta potential and mass deposited when hydrazine is added. The surface of TiN and TiC nanoparticles has been modified to improve the dispersion in isopropyl alcohol adding a cationic polymer (polyethylenimine) as dispersant with two different molecular weights. The influence of acidic/basic character of the solvent also has been evaluated in order to reach the most efficient EPD process. It was found that the adsorption of polyethylenimine with higher molecular weight can preserve the homogeneity of TiC coatings. The surface of TiN nanoparticles can be tuned in order to achieve a similar polyethylenimine adsorption which also improves the deposition in basic media.
\end{abstract}

\section{Introduction}

Transition metal non-oxides have been extensively studied due to their interesting properties. Specially, titanium nitride (TiN) and titanium carbide (TiC) have gained attention due to its attractive properties such as hardness, electrical properties, thermal stability, etc. Surface coatings are the most relevant applications, i.e. improving hardness and wear resistance for surgical tools and implants. Among techniques to process TiN and TiC coatings, such as physical (PVD) or chemical vapor deposition (CVD) or nitridation of $\mathrm{TiO}_{2}$ should be highlighted. PVD includes techniques such as cathodic arc evaporation [1], ion beam deposition [2, 3], ion implantation [4], plasma [5] and laser methods [6, 7]. In those techniques, TiN nanocrystals grow forming typically elongated particles in the range of several hundreds of nanometers and coatings with hardness of approximately $27 \mathrm{GPa}$ are obtained. CVD is often used in the semiconductor industry to obtain TiN thin films via the reaction of metal titanium with nitrogen or ammonia at temperatures above $800^{\circ} \mathrm{C}$ or nanosized titania nitrified in flowing ammonia gas at $1100^{\circ} \mathrm{C}$, being sometimes required the addition of a reducing agent. However, those processes need extremely high pressure and temperature conditions, and production are not massive than requested $[8,9]$. In this sense, the looking for non-expensive methods to shape these hard coatings is still challenging. The electrophoretic deposition (EPD) is a conventional technique that can serve to the purpose of shape non-oxide coatings, but it is limited by the sintering temperatures (over $1800^{\circ} \mathrm{C}$ ) to consolidate the packed particles shaped in a film.

EPD is a colloidal processing method which consists in the electrically driven movement and deposition of charged particles onto a conducting substrate. This technique has received special attention because of its simplicity, low cost, versatility, the possibility to cover complex shapes and an easy scale-up. In EPD, the thickness of the coatings mainly depends on the particle size and the deposit growth depends on the stability and dispersing conditions of the suspensions. Therefore, understanding the colloidal behavior of nanoparticles is a key point in the process. In this work, the characterization of TiN and TiC nanopowders, and its stability in organic suspensions were investigated when a reducing agent as hydrazine is added. First, morphology and crystallography of commercial TiN and $\mathrm{TiC}$ nanopowders were studied. Then, using the more stabilized suspensions, TiN and TiC coatings were obtained by EPD. Finally, the homogeneity of the coatings was 
evaluated as a function of hydrazine concentration and polyethylenimine with different molecular weight.

\section{Experimental}

As-received commercial TiN and TiC nanopowders (Hefei Kaier Nanometer Energy \& Technology, China) were characterized by helium pycnometry (Multipycnometer, Quantachrome Co., USA) to determine its density ( $\rho$ ), and by Physisorption Analyzer (ASAP 2020, Micromeritic, USA) to measure the specific surface. The particle size and the morphology of the powders were analyzed by field emission scanning electron microscopy (FE-SEM) using an S-4700 microscope (Hitachi, Japan). Crystalline phases were characterized by X-Ray Diffraction (XRD) (D8 Advance, Bruker, Germany) using $\mathrm{Cu} K_{\alpha}$ radiation.

TiN and TiC suspensions $\left(0.1 \mathrm{~g} \cdot \mathrm{L}^{-1}\right)$ were prepared using isopropyl alcohol (99.7\%, Panreac, Spain) as solvent and polyethilenimine (PEI, Sigma Aldrich, Germany) as stabilizer. For comparative proposes, 1.5 wt. $\%$ of two PEI with molecular weights of 2,000 and 25,000 g.mol ${ }^{-1}$ (PEI 2,000 and PEI 25,000, respectively) were added to stabilize the suspensions, while hydrazine monohydrate (64-65\%, Sigma-Aldrich, Germany) was used to modify the basicity of the solvent. Mechanical stirring and sonication (Ultrasonication Probe, UP 400S, Hielscher, Germany) were used as dispersing methods to break the soft agglomerates.

The dispersion and stability of TiN and TiC suspensions with 1.5 wt.\% of PEI 2,000 and PEI 25,000 were evaluated as a function of its zeta potential adding different volumes of hydrazine. This parameter was determined by laser Doppler velocimetry using a Zetasizer Nano ZS (Malvern S, UK). Then, TiN and TiC films were shaped from TiN and TiC highly stabilized suspensions (1 g. $\mathrm{L}^{-}$

${ }^{1}$ ) by EPD on as-received stainless steel foils (AISI 304) of 30x20x0.5 mm. The counter electrode was a platinum foil of similar dimensions, separated of the work electrode (ASIS 304) by a distance of $2 \mathrm{~cm}$ in the electrophoretic cell. EPD was performed under galvanostatic conditions with 5 minutes of deposition time using a high voltage power source (2611 System SourceMeter, Keithley Instruments Inc., USA), applying a current density of $50 \mu \mathrm{A} / \mathrm{cm}^{2}$. The film growth was evaluated as a function of the hydrazine concentration.

\section{Results}

The morphology of $\mathrm{TiN}$ and $\mathrm{TiC}$ nanopowders with a relative density of $62.6 \%$ and $78.3 \%$, respectively, were analysed by FE-SEM. As shown in Figure 1, agglomerates of $\sim 200 \mathrm{~nm}$ can be observed for both powders. These agglomerates are formed by nanoparticles of $26-32 \mathrm{~nm}$ to TiN and $41-48 \mathrm{~nm}$ to $\mathrm{TiC}$.
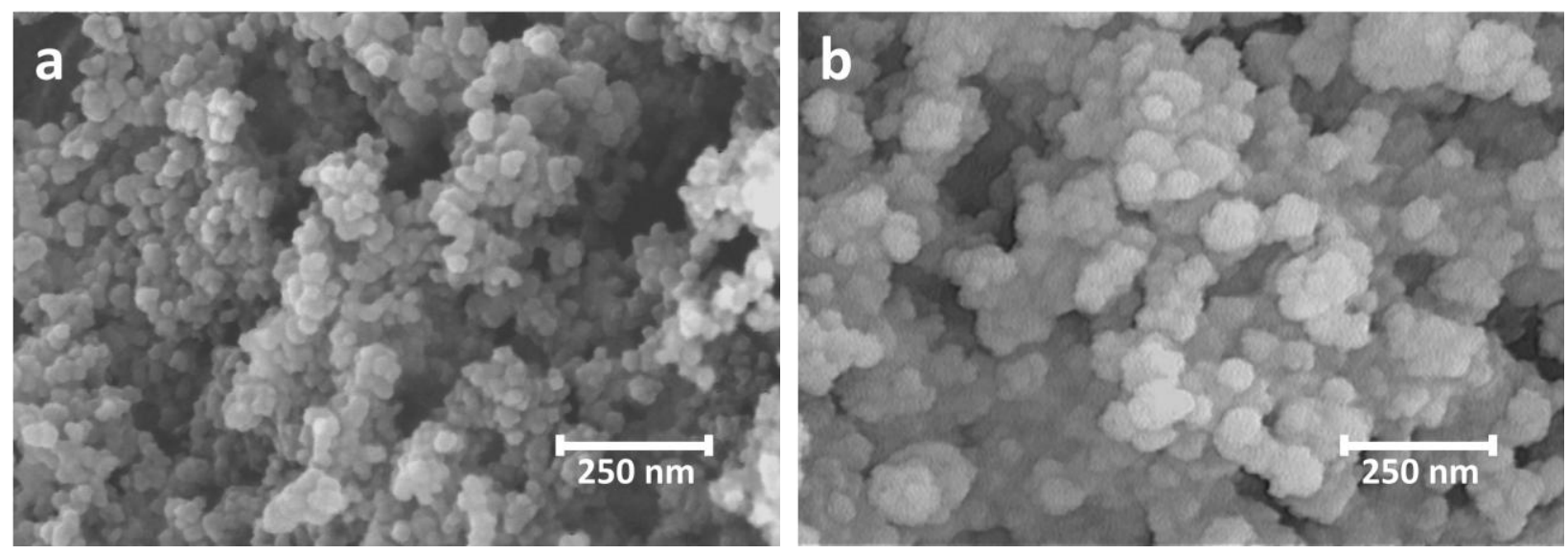

Fig. 1. FE-SEM micrographs of a) TiN and b) TiC nanopowders

X-ray diffraction (XRD) pattern of TiN and TiC nanopowders was also evaluated (not shown). The diffraction pattern indicates the presence of a crystalline phase with three characteristic peaks 
$\left(37.25^{\circ}, 43.20^{\circ}\right.$ and $\left.62.43^{\circ}\right)$ corresponding to (111), (200) and (220) planes of cubic TiN (JCPDS 00-38-1420). XRD peaks of TiC nanopowder with cubic structure were also identified at $36.47^{\circ}$, $42.28^{\circ}$ and $61.05^{\circ}$ (JCPDS No. 00-032-1383).

The stabilization of TiN and TiC suspensions in isopropyl alcohol, prepared using $1.5 \mathrm{wt}$.\% of PEI 2,000 and PEI 25,000 were studied as a function of amount of hydrazine. Figure 2 shows the variation of zeta potential as a function of hydrazine concentration (related to the solid content) for both suspensions.
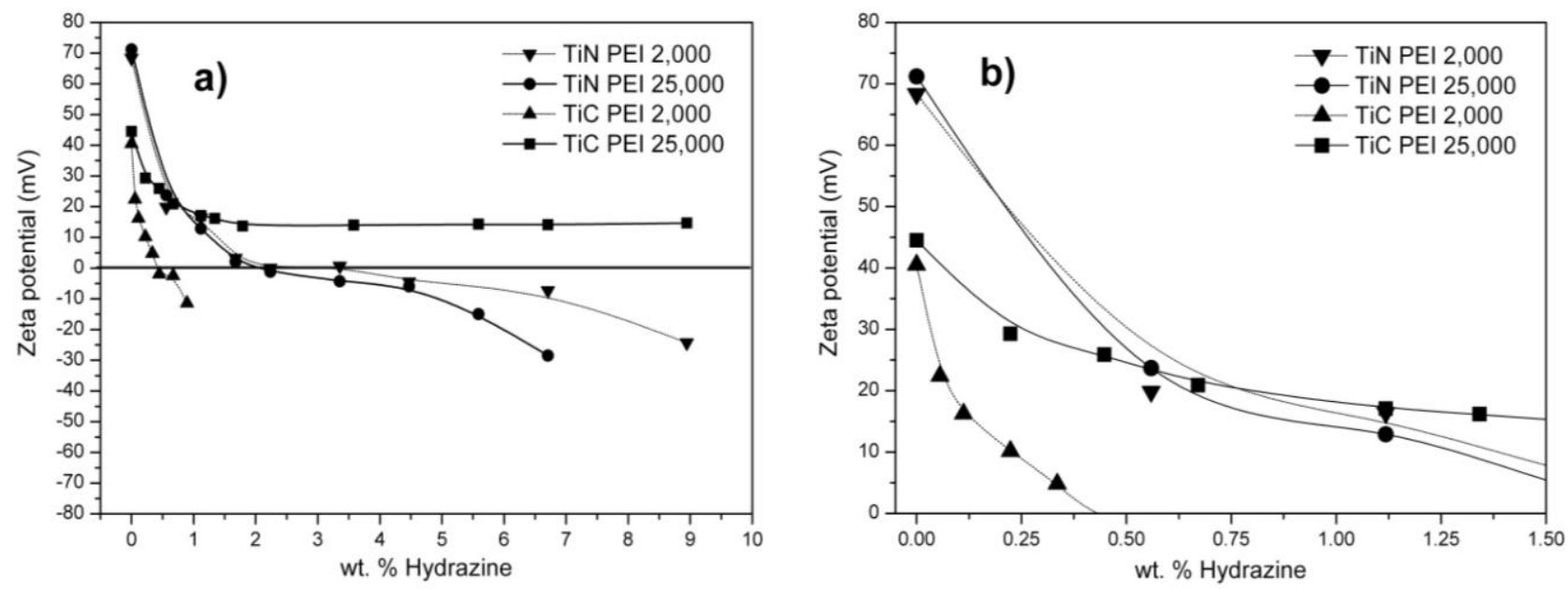

Fig. 2. Evolution of zeta potential with the addition of hydrazine (a) and a zoomed area for the positive zeta potential (b)

Both powders showed negative zeta potentials in isopropyl alcohol $(-10 \mathrm{mV}$ and $-40 \mathrm{mV}$ for TiN and $\mathrm{TiC}$, respectively) evidencing their acidic character. As plot in figure $2 \mathrm{a}$, the addition and adsorption of $1.5 \mathrm{wt}$. \% of PEI leads to positive zeta potentials, $+70 \mathrm{mV}$ for TiN and $+45 \mathrm{mV}$ for $\mathrm{TiC}$, indicating, in all cases a combined electro-steric mechanism of stability to the suspensions. The addition of a reducing agent as hydrazine modifies the ionization state of PEI, which is adsorbed on the surface of nanoparticles. For both powders and both PEIs, the zeta potential decreasing shows a reduction of the electrostatic component of the electro-steric mechanism of stabilization provided by the PEI addition.

Independently of the chain length of PEI, the values of zeta potential for $0 \mathrm{wt} . \%$ of hydrazine were similar for TiN. Similar behaviour is observed for TiC nanoparticles. This effect also occurs using TiC nanoparticles. However, absolute values differ from one powder to the other. Both powders exhibit specific surface areas in the same order of magnitude, $44.42 \mathrm{~m}^{2} \mathrm{~g}^{-1}$ for TiN and $31.65 \mathrm{~m}^{2} \mathrm{~g}^{-1}$ for TiC. PEI adsorbs in acidic sites, so when a similar amount of PEI is added to disperse TiN and $\mathrm{TiC}$, the neutralization of the surface leads dissimilar charge states for each powder, corresponding with their differences in the acidic character. The addition of hydrazine neutralises the adsorbed PEI chains and the zeta potential decreases, except to TiC dispersed with PEI 25,000, which maintains positive.

The influence of the incorporation of hydrazine to the nanoparticles suspension and the effect in the homogeneity of the coatings were studied by cathodic electrophoretic deposition. In this sense, only suspensions prepared in the range of zeta potential plotted in figure $2 b$, were considered for these tests. EPD was carried out applying a current density of $50 \mu \mathrm{A} / \mathrm{cm}^{2}$ for a constant deposition time of $5 \mathrm{~min}$. Figure 3 shows the deposited mass of TiN and TiC stabilised suspensions with 1.5 wt. \% of PEI 2,000 and PEI 25,000 as a function of hydrazine amount. 


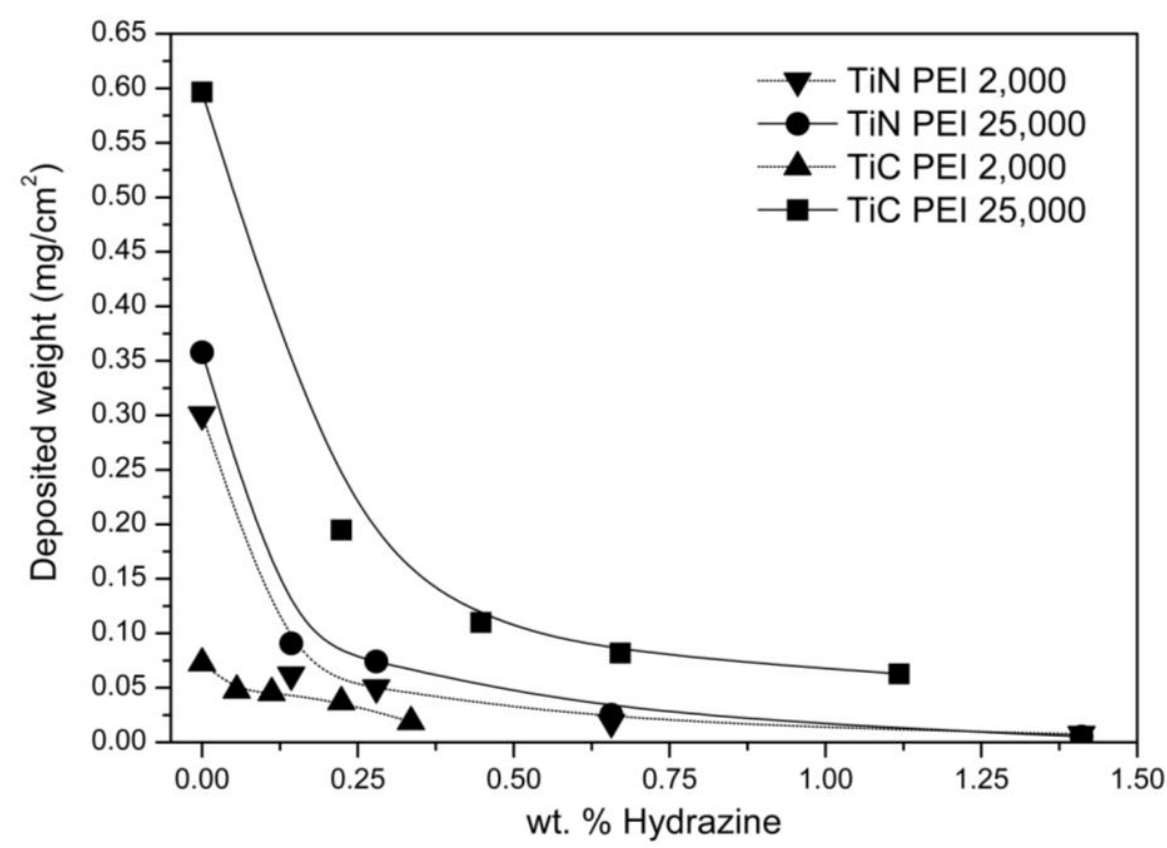

Fig. 3. Evolution of deposited mass by EPD with increasing concentration of hydrazine

The increment of hydrazine concentration decreases the deposited mass and the homogeneity of the coatings. The decrease of mass deposited is mainly associated to the reduction of the particles mobility associated with the addition of the reducing agent.

Figure 4 shows the TiN layers prepared with PEI 25,000 when the concentration of hydrazine increases. Homogeneous coating is only obtained for $0 \mathrm{wt} \%$ hydrazine. The addition of hydrazine leads to the appearance of heterogeneities in the coatings which are more evident with the maximum amount of hydrazine. Similar behaviour was observed for PEI 2,000 (not shown).

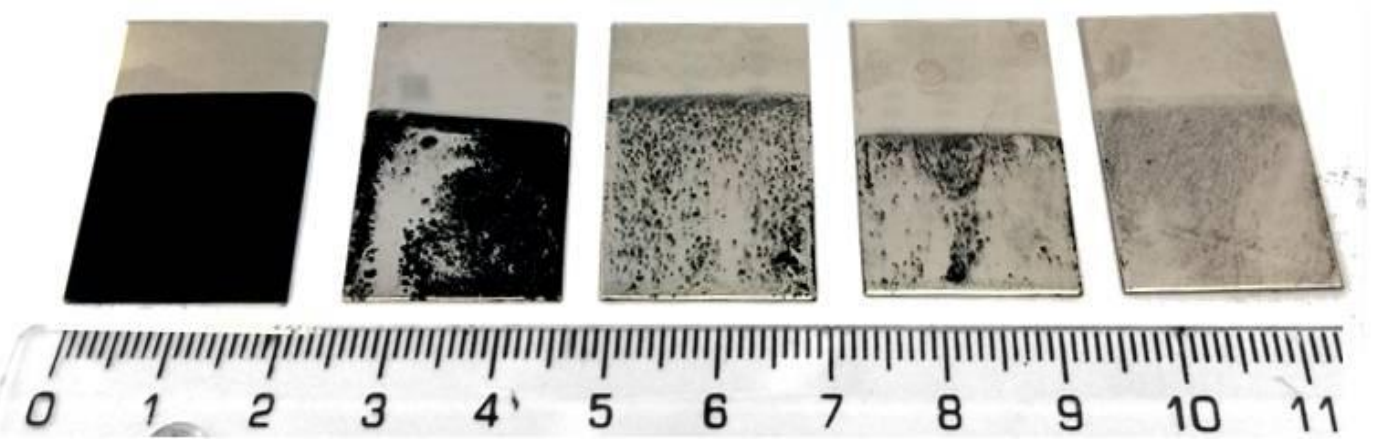

Fig. 4. TiN coatings as a function of hydrazine using PEI 25,000.

Figure 5 shows the TiC coatings obtained using PEI 2,000 and 25,000. TiC nanoparticles have a completely different behaviour depending on the chain length of the polymer. In this way, when PEI 25,000 is used to stabilise the TiC nanoparticles and hydrazine is added, the deposition kinetics decrease but the homogeneity of the coatings was maintained. In the case of PEI 2,000, heterogeneities in the coatings appear from the first addition of hydrazine. Consequently, the adsorption of a long chain can avoid the deterioration of the coatings when the acidic/basic character of the solvent change. However, the amount and the suitable conditions to use PEI 25,000 should be adjusted and tuned to provide similar stable conditions for the TiN nanoparticles than those reached for the $\mathrm{TiC}$ nanoparticles. 


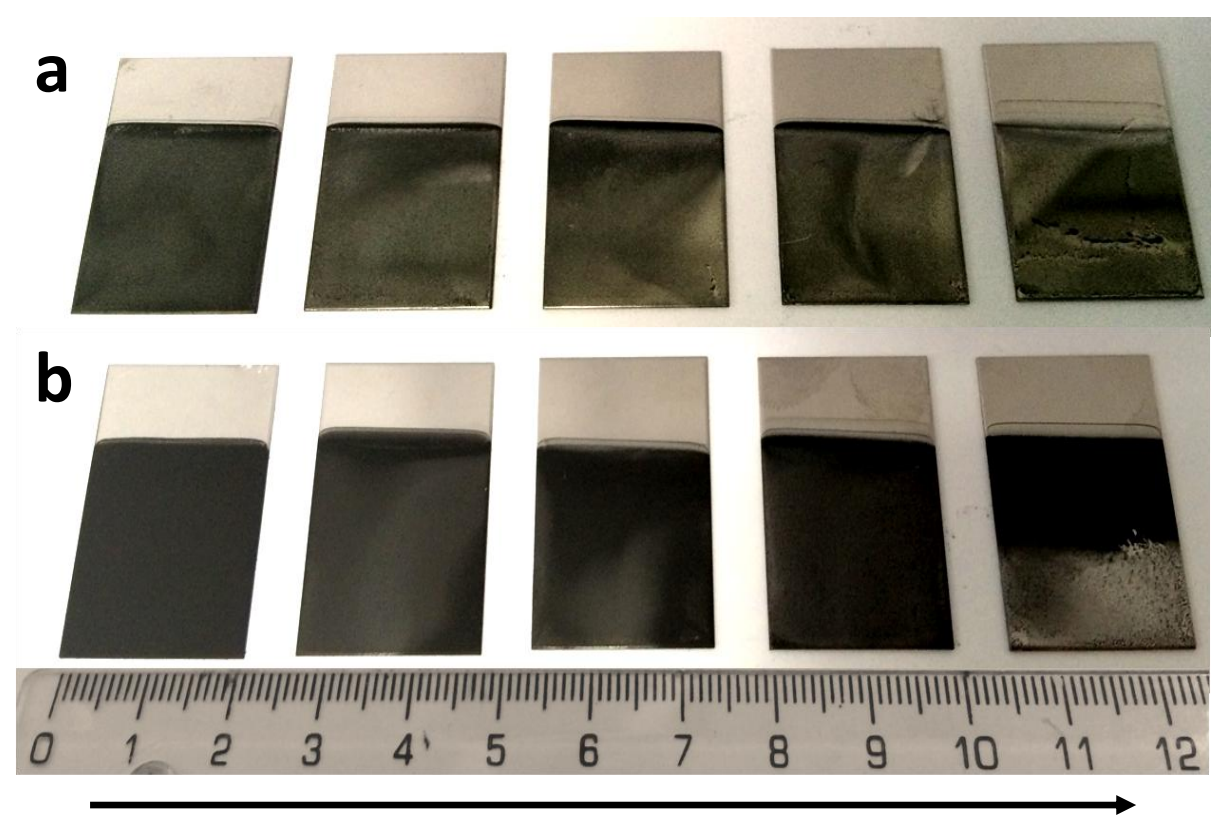

Fig.5. TiC coatings with increasing concentrations of hydrazine in stabilized suspensions using PEI 2,000 (a) and PEI 25,000 (b)

\section{Conclusions}

Optimal dispersion conditions of TiN and TiC nanoparticles were achieved using PEI with different molecular weight. PEI adsorption provides a high repulsive electro-steric interaction between nanoparticles suspended in isopropanol. Using these suspensions, stainless steel substrates were completely and homogeneously covered by a TiC and TiN layer.

Electrophoretic deposition was evaluated in terms of homogeneity of the coating, when the acidic/basic character of the solvent change by adding a strong reducing agent, as hydrazine. Homogeneous TiN coatings were only obtained for $0 \mathrm{wt} \%$ of hydrazine, while TiC coatings maintained the homogeneity up to $0.7 \mathrm{wt} . \%$ of hydrazine. Results show that the adsorption of PEI with higher molecular weight can preserve the homogeneity of the TiC coatings, evidencing the possibility to tune the TiN surface in order to achieve a similar PEI adsorption which also improves TiN deposition in basic media. Thus, EPD appears as a suitable method to obtain homogeneous TiN and TiC coatings through the manipulation of the electrical charges at the surface of nanoparticles.

\section{References}

[1] L. A. Dobrzaski and K. Golombek, Materials Research, 8, (2005), 113-116

[2] N. B. Dahotre, P. Kadolkar and S. Shah, Surface and Interface Analysis, 31, (2001), 682-691

[3] R. A. Andrievski, Uspekhi Khimii, 74, (2005), 1163-1175

[4] A. Meldrum, L. A. Boatner and C. W. White, Nuclear Instruments and Methods in Physics Research, Section B: Beam Interactions with Materials and Atoms, 178, (2001), 7-16

[5] K. H. Kim and S. H. Lee, Thin Solid Films, 283, (1996), 165-170

[6] S. V. Petrov, Powder Metallurgy and Metal Ceramics, 36, (1997), 483-486

[7] P. R. Willmott and J. R. Huber, Reviews of Modern Physics, 72, (2000), 315-328

[8] S. Kawano, J. Takahashi and S. Shimada, Journal of the American Ceramic Society, 86, (2003), 1609-1611

[9] J.-G. Li, L. Gao, Q.-H. Zhang, et al., Wuji Cailiao Xuebao/Journal of Inorganic Materials, 18, (2003), 765-771 